\title{
Doença vascular periférica acima e abaixo do diafragma A equipe médica e o intervencionista devem atuar juntos?
}

\author{
Vascular and cardiovascular disease: \\ the multidisciplinary heart and interventional team
}

\section{Carlos Eduardo Virgini-Magalhães*}

Esmeralci Ferreira

\section{Resumo}

Atualmente o conceito de equipe multidisciplinar tem sido muito enfatizado como uma grande ferramenta na abordagem de pacientes com doenças cardiovasculares. A introdução de tratamentos com uso de técnicas percutâneas menos invasivas, como opção ao tradicional tratamento cirúrgico abriu novos horizontes para ampliação de divisão de responsabilidades entre os especialistas. Esta discussão, envolvendo intervencionistas, cirurgiões e clínicos, possibilitou uma disseminação que foi de extrema utilidade, não somente para a prática clínica diária, como também possibilitou a elaboração de estudos clínicos randomizados, registros e diversos protocolos utilizados nas rotinas institucionais.

A atuação conjunta propicia uma grande segurança na tomada de decisão para um determinado tipo de tratamento com maior benefício para subgrupos de alto risco. A experiência da equipe multidisciplinar associada às evidências $\mathrm{e}$ recomendações pautadas na literatura fortalece este conceito. A despeito da inexistência de estu- dos robustos que apoiem melhores resultados na rotina deste fórum de discussões, há uma forte inclinação para o fortalecimento desta forma de conduta. O objetivo deste artigo é discutir aspectos relevantes da equipe multidisciplinar na otimização das decisões no que tange ao tratamento das doenças vasculares periféricas acima e abaixo do diafragma.

Descritores: Doença arterial periférica; Procedimentos endovasculares; Equipe de assistência ao paciente; Estenose carotídea; Aorta; Gangrena.

\begin{abstract}
Currently the concept of multidisciplinary team has been much emphasized as a major tool in the management of patients with cardiovascular disease. The introduction of treatments with the use of less invasive percutaneous techniques, with options to traditional surgery has opened new horizons for expanding division of responsibilities among experts. This discussion involving interventional surgeons and clinicians provided a spread that was extremely useful,
\end{abstract}


not only for the daily clinical practice, but also enabled the development of randomized clinical trials, registries and various protocols used in institutional routines.

The joint efforts provide a great safety in decision making for a particular type of treatment and which is most effective for high-risk subgroups. The experience of the multidisciplinary team, coupled with the evidence and recommendations guided by the literature strengthens this concept. Despite the lack of robust studies supporting better outcomes in routine discussions of this forum there is a strong tendency to strengthen this type of conduct. The purpose of this article is to discuss relevant aspects of the multidisciplinary team in optimizing the decisions regarding the treatment of peripheral vascular disease above and below the diaphragm.

Keywords: Peripheral arterial disease; Endovascular procedures; Multidisciplinary team; Carotid stenosis; Aorta; Gangrene.

\section{Introdução}

As doenças vasculares envolvendo as regiões supra e infradiafragmáticas eram historicamente tratadas com cirurgia. A partir da introdução dos procedimentos endovasculares percutâneos, os intervencionistas têm tratado um número cada vez maior de pacientes utilizando endopróteses. Em que pese esta ampla disseminação de procedimentos diferentes da clássica cirurgia, a estratégia na escolha deve ser baseada na complexidade da lesão, no perfil clínico do paciente, na experiência do operador no manuseio dos dispositivos e na disponibilidade destes materiais. O papel do operador nesta tomada de decisão é de suma importância, entretanto a escolha da terapêutica a ser instituída depende igualmente do clínico, cuja responsabilidade na condução do caso se reflete no conhecimento prévio de todos os fatos relevantes à condição do paciente.

$\mathrm{O}$ correto esclarecimento sobre riscos e benefícios das formas possíveis de tratamento deve ser a tônica nas entrevistas com os pacien- tes. Um linguajar acessível, que se enquadre socioculturalmente a cada indivíduo, deve ser exaustivamente estabelecido na relação entre o operador e o paciente. A moderação do clínico, permitindo que se esclareçam detalhes que possam ter sido omitidos, intencionalmente ou não, evita vieses de seleção de um determinado modelo de abordagem. O aconselhamento do paciente, orientado pela equipe multidisciplinar, permite ponderar prós e contras nas diferentes estratégias, evitando que se crie uma tendência inadequada para um tratamento específico. Não obstante a simplicidade teórica destas medidas, é fundamental que a preferência da conduta seja feita em bases estabelecidas para cada paciente.

A análise individual é o maior fator relacionado ao sucesso do procedimento. Neste processo, a ponderação do clínico se faz necessária. A habilidade técnica do operador, a escolha do material e o ambiente hospitalar são importantes, mas dependem do que a análise multidisciplinar decidiu para aquele paciente, especificamente. Um exemplo claro está naqueles indivíduos idosos, cuja morbimortalidade cirúrgica pode ser proibitiva, orientando para uma abordagem percutânea. Nem sempre estas decisões se fazem de forma fácil, principalmente no que se refere ao alto custo das próteses intravasculares, pois independente do fato de serem custo-efetivas na maioria dos cenários, os recursos públicos raramente contemplam a sua utilização. Na realidade, mesmo no ambiente de saúde suplementar há certa dificuldade na adesão a estas tecnologias.

A ênfase para a conduta individualizada do clínico no padrão de encaminhamento à equipe multidisciplinar se faz necessária para nortear todos estes aspectos, inclusive éticos. Em um país de proporções continentais, até mesmo diferenças regionais na condução de cada caso podem ser mais bem avaliadas pelo generalista. Além disso, médicos com diferentes experiências às vezes são discordantes na tomada de decisão. Uma perfeita indicação somente será obtida com trabalho conjunto, consenso, despojamento de vaidades e foco no 
bom resultado para o paciente. Esta equipe tem de ter em mente que o processo de decisão que leva ao aconselhamento ao paciente em nenhum momento pode substituir a relação individual (médico-paciente) que se traduz na única maneira de sabermos todos os anseios dos pacientes para que o mesmo possa participar, como maior interessado, da decisão final de seu tratamento. O respeito a estas escolhas deve ser considerado.

\section{Aspectos históricos}

Hoje o que se denomina de equipe multidisciplinar para tomada de decisões não é um conceito novo na medicina. A arte de ensinar dos mestres da deontologia médica já postulava, na ciência do dever e da obrigação, um mosaico de ensinamentos sobre as escolhas éticas e morais do que deve ser feito. A associação do conhecimento médico, alicerçado na experiência e educação continuada é e sempre foram passados a cada nova geração de estudantes de medicina nas enfermarias dos hospitais universitários, santas casas e instituições de ensino. A diferença fundamental para os dias de hoje é que as equipes multidisciplinares, na maioria das vezes, necessitam da participação de subespecialidades cada vez mais inseridas em decisões terapêuticas complexas. Mesmo estabelecendo seu funcionamento na informalidade, as decisões multidisciplinares são uma realidade bastante conhecida e utilizada.

Um claro divisor de águas no estabelecimento oficial destas equipes se deu por intermédio da American Heart Association, ${ }^{1}$ quando em 1995 orientou a criação de uma equipe multidisciplinar na avaliação dos pacientes portadores de insuficiência cardíaca grave, para decisão da forma de tratamento médico, cirúrgico ou de transplante cardíaco. A partir daí a utilização deste conceito de foi reproduzida forma organizada em diversos estudos randomizados e enquadrada na prática clínica diária de diversas instituições. No campo científico da cardiologia, um dos mais produtivos estudos utilizando equipe multidisciplinar foi o estudo SYNTAX. ${ }^{2}$ Este estudo randomizado foi o primeiro a incluir pacientes com doença coronariana multiarterial complexa, incluindo lesões em tronco de coronária esquerda. O julgamento sobre a possível realização de procedimento através de cirurgia de revascularização ou intervenção percutânea com stent farmacológico era feito pelo cirurgião cardíaco e pelo cardiologista intervencionista. Os pacientes elegíveis para ambas as formas de tratamento eram randomizados e aqueles que tinham contraindicação estabelecida pelo especialista para uma das formas de tratamento eram incluídos em um registro.

\section{Aspectos práticos}

Independentemente do que o estudo SYNTAX representou para o estabelecimento desta prática, temos que reconhecer que no "mundo real" a atuação da equipe multidisciplinar é bastante diferente dos ensaios randomizados nos quais se procura estabelecer um padrão de tratamento para uma pergunta postulada e ainda sem resposta nas recomendações e evidências. $\mathrm{O}$ que se procura analisar como melhor forma de tratamento no mundo real é a utilização das diretrizes orientadas pelas sociedades científicas. Para as recomendações em que o nível de evidência não deixa margem de dúvida, as equipes multidisciplinares apenas corroboram a escolha, sem necessidade de debate. A utilização de protocolos baseados nestas diretrizes e o consenso dos participantes podem trazer grandes benefícios na aplicação dos mesmos nas instituições, melhorando resultados e evitando decisões isoladas do especialista. Além das diretrizes, a inclusão de escores de risco pode ser uma ferramenta útil na avaliação dos pacientes. Mas, a despeito da aplicabilidade de escores de risco, ainda temos que realizar a avaliação clínica do paciente, no que se refere a idade, sexo, índice de massa corporal, assim como comorbidades tais como a doença vascular periférica, a insuficiência renal, a disfunção ventricular esquerda, o diabetes e o histórico pregresso de acidente vascular encefálico (AVE), o infarto do miocárdio e a cirurgia cardíaca. Todos estes fatores têm que ser ponderados e frequentemente 
temos que agregar outros especialistas do tipo nefrologistas, geriatras, neurologistas e oncologistas. A expectativa de vida dos pacientes é um dos aspectos mais relevantes para a equipe de profissionais e a tomada de decisões.

No que tange ao tratamento das doenças vasculares periféricas acima e abaixo do diafragma, a equipe de médicos e intervencionistas devem atuar juntos, pois a complexidade da maioria destes pacientes exige mais de uma técnica de atuação. O clínico, o cirurgião, o intervencionista e $\mathrm{o}$ anestesista precisam ser atuantes na escolha da melhor técnica e atribuição de tática para cada indicação de intervenção. A escolha e disponibilização de materiais do tipo endopróteses, introdutores, guias metálicos norteiam um resultado de sucesso. A necessidade de retaguarda cirúrgica in loco para eventuais complicações é mandatória.

Muitos dos procedimentos intervencionistas em cardiologia e/ou radiologia vascular não podem prescindir da participação de métodos de imagem durante o procedimento. A necessidade de ecocardiograma, ultrassonografia, ressonância magnética são fundamentais na boa indicação do procedimento e controle de resultados. A disponibilidade de profissionais de enfermagem experientes e familiarizados com os dispositivos, assim como uma equipe de tecnólogos em radiologia são importantes pilares de suporte ao procedimento.

\section{Colaboração no cuidado do paciente vascular}

$\mathrm{O}$ emprego de equipes multidisciplinares está bem estabelecido no tratamento das doenças malignas. ${ }^{3}$ Esta prática foi desenvolvida para garantir aos pacientes os melhores padrões de práticas baseadas em evidências. Hoje há o reconhecimento implícito de que o tratamento do câncer é mais efetivo quando realizado por uma equipe multidisciplinar que inclua cirurgiões, oncologistas, radiologistas e enfermeiras. O tratamento do câncer é certamente melhor realizado através de reuniões regulares, nas quais casos individuais são discutidos, a abordagem planejada e qualquer variação de tratamento é explicada e acordada.

As necessidades da cirurgia vascular moderna são idênticas. A doença vascular periférica é melhor manejada por um time que inclui o cirurgião vascular, o clínico, o anestesista, tecnólogos e a enfermagem especializada. Esta equipe deve habitualmente ser suplementada por membros de outras disciplinas médicas incluindo intensivistas e endocrinologistas, entre os mais frequentes. ${ }^{4} \mathrm{~A}$ prática colaborativa deve ir além e envolver atividades educacionais combinadas, treinamento cruzado entre membros de cada equipe, e idealmente as visitas ambulatoriais e a estrutura de intervenção deve ser única ou, pelo menos, integrada. ${ }^{5}$

O próprio diagnóstico da doença arterial obstrutiva periférica (DAOP) precisa ser estimulado: o estudo PARTNERS mostrou que $29 \%$ dos pacientes estudados eram portadores de DAOP mas apenas $49 \%$ dos médicos tinham conhecimento deste diagnóstico nos seus pacientes. ${ }^{6}$

\section{Principais síndromes e sua interface clínica}

As principais síndromes clínicas associadas às lesões obstrutivas periféricas e acessíveis ao tratamento vascular são a isquemia cerebral de origem extracraniana causada pelas estenoses da bifurcação carotídea e a doença arterial obstrutiva periférica propriamente dita, com seus diferentes níveis de obstruções ateroscleróticas que determinam a redução da perfusão para os membros inferiores e que podem resultar em amputação dos membros.

\section{Estenose carotídea}

O acidente vascular cerebral (AVC) é a terceira causa de óbito e a primeira causa de invalidez nos Estados Unidos. Algo entre 500 e 600 mil novos AVCs isquêmicos ocorrem a cada ano neste país. Em teoria cerca de $250 \mathrm{mil}$ casos poderiam ser evitados através da correção cirúrgica de estenoses carotídeas.

A vasta maioria dos indivíduos com doença 
importante de artéria carótida é completamente assintomática, sendo identificada pela presença de um sopro cervical. O AVC pode ser a primeira manifestação clínica da estenose carotídea. Uma minoria de pacientes com doença importante de artéria carótida pode ser identificada após procurar tratamento depois de um ataque isquêmico transitório (AIT). O quadro clínico mais comum é conhecido como amaurosis fugax (perda aguda total ou parcial da visão em um olho com poucos minutos de duração), mas qualquer déficit neurológico focal que reverta completamente em até 24 horas é considerado um AIT.

Em pacientes assintomáticos com uma estenose importante de artéria carótida interna, o risco de AVC durante os 5 anos subsequentes é de aproximadamente $10 \%$ a $15 \%$. Por outro lado, em pacientes que experimentaram um AIT, a incidência de AVC é de, aproximadamente, 25\% dentro do primeiro ano, embora a maioria ocorra precocemente, dentro das primeiras semanas ou meses depois do AIT.

A eficácia da endarterectomia carotídea (EC) para o tratamento da estenose de artéria carótida foi confirmada no começo dos anos 1990 por grandes estudos multicêntricos que compararam a cirurgia com o tratamento clínico, incluindo o North American Symptomatic Carotid Endarterectomy Trial (NASCET) e o Asymptomatic Carotid Atherosclerosis Study (ACAS). A partir do desenvolvimento das técnicas endovasculares a angioplastia de carótida foi proposta como possível alternativa à endarterectomia.

\section{Endarterectomia $\mathrm{x}$ angioplastia}

Há pelo menos uma década se discute as duas técnicas de tratamento das estenoses carotídeas (endarterectomia $\mathrm{x}$ angioplastia) e, a despeito do grande volume de estudos prospectivos e randomizados publicados na literatura, este tema permanece controverso e é objeto de intenso debate nos dias atuais. Pelo menos 5 diretrizes respaldadas por grandes sociedades científicas foram publicadas recentemente com recomendações, algumas vezes discordantes, sobre a indicação dos 2 métodos de tratamento. ${ }^{7}$

A maior parte destas diretrizes recomenda a endarterectomia de carótida em pacientes sintomáticos com pelo menos $70 \%$ de estenose carotídea e bom risco cirúrgico. A angioplastia deve ser preferida à cirurgia em pacientes com traqueostomias, irradiação cervical prévia, estenoses localizadas no nível ou acima do corpo vertebral de $\mathrm{C} 2$, ou outras situações consideradas como pescoço hostil. A angioplastia também deve ser preferida em pacientes sintomáticos com pelo menos $50 \%$ de estenose carotídea e alto risco cirúrgico cardiovascular como a presença de doença coronariana grave não passível de correção ou insuficiência cardíaca congestiva. ${ }^{7}$

O tratamento de escolha para pacientes assintomáticos permanece incerto. Grandes estudos multicêntricos demonstraram que a endarterectomia e a angioplastia estão associadas a evoluções similares em assintomáticos. Por outro lado, estudos recentes indicam que apenas o tratamento clínico otimizado (sem intervenção) é suficiente para o controle da maioria das estenoses carotídeas assintomáticas.?

\section{Interface na tomada de decisão para estenose carotídea}

Por se tratar de assunto ainda controverso ,a indicação cirúrgica e a escolha da técnica de intervenção nas estenoses carotídeas poderia se beneficiar de uma equipe multidisciplinar para tomada de decisão. A interlocução entre cirurgião vascular, neurologista, cardiologista, entre outras especialidades clínicas, pode oferecer visões diferentes e complementares sobre cada caso individual, maximizar a conduta e reduzir a exposição de muitos pacientes ao risco de uma intervenção desnecessária. O National Institute for Health and Clinical Excellence, do Reino Unido, recomenda que a seleção de pacientes deve ser realizada por um time multidisciplinar e sugere que a equipe multidisciplinar deve se reunir frequentemente para discutir todas as intervenções carotídeas e provavelmente todos os exames alterados de carótidas. ${ }^{8} \mathrm{O}$ documento 
explicita ainda que em unidades que realizam intervenção em pacientes assintomáticos a garantia e mútuo apoio da equipe multidisciplinar é essencial, em particular para assegurar que as boas práticas clínicas sejam consideradas como possível alternativa. No Reino Unido, a angioplastia de carótida não está bem estabelecida, como é o caso da endoprótese de aorta abdominal, mas sua introdução em um centro através de uma equipe multidisciplinar deve avalizar que pacientes não sejam submetidos a riscos desnecessários. ${ }^{9}$

\section{Doença arterial obstrutiva periférica}

A doença arterial obstrutiva periférica (DAOP) se caracteriza pela obstrução aterosclerótica progressiva das artérias periféricas, especialmente as dos membros inferiores, afetando gradualmente e de forma adversa a qualidade de vida destes pacientes. Estudos populacionais identificaram que aproximadamente $20 \%$ dos indivíduos com mais de 60 anos têm algum grau de DAOP. A incidência é também elevada entre fumantes, diabéticos e em pacientes com doença arterial coronariana. ${ }^{4} \mathrm{Em}$ reconhecimento ao surgimento da DAOP como um problema de saúde significativo o Department of Health and Children do governo irlandês produziu, em 2010, um guia médico geral para DAOP para divulgação dentro do sistema público de saúde daquele país. ${ }^{10}$

A DAOP representa um importante marcador de aterosclerose sistêmica e do risco de complicações cardiovasculares e cerebrovasculares, como o infarto agudo do miocárdio (IAM) e o AVC. A despeito do reconhecimento da DAOP como preditor de eventos isquêmicos, esta expressão da aterosclerose acessível à história e ao exame físico é pouco pesquisada pelos clínicos. O diagnóstico precoce da DAOP oferece uma oportunidade única de atuação sobre os principais fatores de risco e modificação do perfil cardiovascular, melhorando, assim, a mortalidade e a qualidade de vida destes pacientes. Nos últimos anos, mudanças importantes na utilização de métodos diagnósticos e no desenvolvimento de técnicas endovasculares vêm modificando e ampliando significativamente a prática dos profissionais envolvidos no tratamento da DAOP.

\section{História natural da DAOP}

A maioria dos pacientes com diagnóstico de DAOP se apresenta com claudicação intermitente ou é assintomática. Outra parcela significativa apresenta dor em membros inferiores pouco característica e apenas um pequeno grupo de pacientes sofre de isquemia crítica do membro. Com o passar do tempo os sintomas se tornam estáveis na maioria dos pacientes. Após 5 anos cerca de $20 \%$ dos indivíduos claudicantes referem piora dos sintomas e entre 5 e $10 \%$ de pacientes desenvolvem isquemia crítica. ${ }^{11}$ Por outro lado, eventos cardiovasculares (IAM e AVE) ocorrem em cerca de 1/3 destes indivíduos e são a principal causa de mortalidade nesta população.

\section{Isquemia crítica do membro}

Uma pequena parcela dos pacientes com DAOP se apresenta ao diagnóstico com isquemia crítica (IC) do membro ou desenvolve a IC ao longo da evolução da DAOP. A IC do membro está associada a uma elevada morbimortalidade no médio prazo. Pacientes com IC têm 3 a 5 vezes mais chances de morte por causa cardiovascular do que não portadores de DAOP. Após 1 ano do diagnóstico de IC, menos da metade dos pacientes estará viva e livre de amputações, $30 \%$ terá sofrido algum tipo de amputação de membros e $25 \%$ terá morrido. Após 5 anos apenas metade destes enfermos estará viva. ${ }^{12}$

A IC é caracterizada pela dor em repouso e/ou pela presença de lesão trófica do membro. A dor costuma ser contínua, mais importante à noite, só aliviando com o membro pendente ou com o uso de analgésicos opiáceos em casos mais graves. A úlcera isquêmica e a gangrena representam o estágio final da DAOP e significam, na prática clínica, a iminência de amputação do membro isquêmico.

Os fatores de risco para desenvolver IC 
do membro incluem idade acima de 65 anos, diabetes mellitus, dislipidemia, tabagismo, entre outros. Sem dúvida, os mais importantes fatores de risco são o diabetes e o tabagismo. $\mathrm{O}$ diabetes aumenta o risco de IC em 4 vezes e o tabagismo triplica este risco. Pacientes diabéticos com IC têm um risco 10 vezes maior de amputação do que não diabéticos. Cerca de 1 membro é amputado a cada 30 segundos por causa do diabetes. ${ }^{13}$

\section{Doença obstrutiva aortoilíaca}

A doença aterosclerótica oclusiva envolvendo a aorta infrarrenal e as artérias ilíacas é uma causa comum de insuficiência arterial sintomática dos membros inferiores. A obstrução aortoilíaca pode ser diagnosticada pela presença de claudicação glútea, de quadril e de coxas, ou ainda pela ausência de pulsos femorais. A tríade clássica de claudicação, impotência sexual e ausência de pulsos femorais caracteriza a chamada síndrome de Leriche.

Embora o controle dos fatores de risco seja mandatório nestes pacientes, a maioria dos cirurgiões tende a indicar cirurgia precocemente para estas lesões. Devido ao grande calibre e ao fluxo sanguíneo intenso dos vasos, o território aortoilíaco possui os melhores resultados das cirurgias de revascularização. Seja por cirurgia aberta ou pela técnica endovascular, as altas taxas de patência no longo prazo autorizam a intervenção precoce neste território, mesmo em pacientes claudicantes, desde que se observe o bom senso. Em cerca de metade dos casos a doença aortoilíaca coexiste com a doença oclusiva infrainguinal, mas na maioria das vezes o reparo das obstruções proximais oferece alívio aos sintomas clínicos.

\section{Cirurgia convencional $\mathrm{x}$ endovascular}

A indicação e a escolha do método de reparo cirúrgico devem levar em consideração a intensidade dos sintomas, o risco operatório e a anatomia arterial. De modo geral, apenas a isquemia crítica e a chamada claudicação incapacitante devem ser alvo de uma terapia intervencionista, seja ela tradicional, seja minimamente invasiva. A literatura especializada mostra que não há diferenças no longo prazo quando comparamos o tratamento conservador adequado com a intervenção cirúrgica em pacientes claudicantes. ${ }^{14}$

Nos últimos anos, o desenvolvimento das técnicas endovasculares reduziu significativamente os riscos inerentes ao procedimento cirúrgico convencional de revascularização do território aortoilíaco. O resultado desta inovação tecnológica foi a ampliação do número de pacientes beneficiados, em especial indivíduos claudicantes, pois tornou-se mais fácil e mais seguro operar estes pacientes. Atualmente, a técnica endovascular quase sempre é a primeira escolha para a intervenção no território aortoilíaco e as indicações para a cirurgia convencional vêm se tornando cada vez mais restritas a lesões obstrutivas graves e aos casos de falha do procedimento endovascular.

O Inter-Society Consensus for the Management of Peripheral Arterial Disease (TASC II), ${ }^{13}$ publicado em 2007 em sua segunda edição, classifica as lesões aortoilíacas e define que nas lesões TASC A a técnica endovascular é a intervenção de escolha, enquanto as lesões TASC D devem ser tratadas por cirurgia aberta através da confecção de derivação cirúrgica ou endarterectomia. Nas lesões TASC B, a técnica endovascular é o tratamento preferencial e a cirurgia aberta é a preferida nas lesões tipo C em pacientes com bom risco cirúrgico (figura 1 e tabela 1 ).

\section{Obstrução femoropoplítea}

O segmento femoropoplíteo é o mais acometido pela doença obstrutiva aterosclerótica. Os vasos primariamente envolvidos são as artérias femoral superficial e poplítea. Devido a sua cronicidade, boa parte dos indivíduos com oclusões extensas de femoral superficial pode apresentar doença assintomática ou referir queixas leves de dor em panturrilhas, caracterizadas ou não como claudicação. Frequentemente, devido ao longo período de instalação e cresci- 
Tabela 1. Lesões passíveis de reconstrução cirúrgica aberta no território aortoilíaco conforme a classificação TASC

Lesões de tratamento preferencial (tipo C) e de tratamento de escolha (tipo D) por cirurgia aberta.

\section{Lesões tipo C}

- Oclusões bilaterais de ilíaca comum;

- Estenoses bilaterais entre 3-10 cm de extensão que não se continuam pela femoral comum;

- Estenose unilateral de ilíaca externa que se estende à femoral comum;

- Oclusão unilateral de ilíaca externa que envolve a origem da hipogástrica e/ou da femoral comum;

- Oclusão de ilíaca externa unilateral muito calcificada com ou sem envolvimento da origem da hipogástrica e/ou da femoral comum.

\section{Lesões tipo D}

- Oclusão aortoilíaca infrarrenal;

- Doença difusa envolvendo a aorta e ambas as ilíacas necessitando tratamento;

- Estenoses múltiplas difusas envolvendo a ilíaca comum, externa e femoral comum unilateral;

- Oclusão unilateral de ambas as ilíacas comum e externa;

- Oclusão bilateral da ilíaca externa;

- Estenoses ilíacas em pacientes com AAA necessitando tratamento e não candidatos a endoprótese ou outras lesões que necessitem cirurgia aortoilíaca aberta.

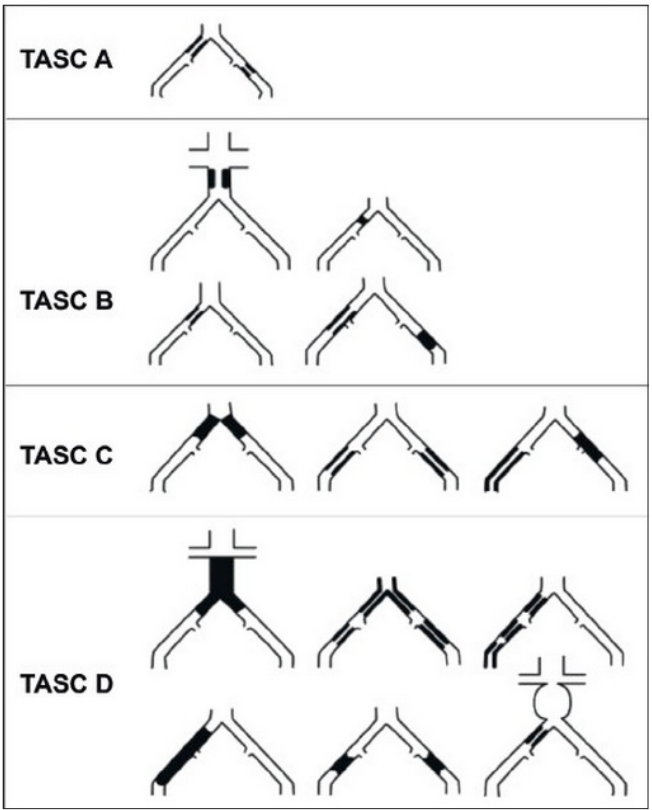

Figura 1. Classificação (esquema) das lesões aortoilíacas segundo o TASC II
Tabela 2. Lesões passíveis de reconstrução cirúrgica aberta no território infrainguinal conforme a classificação TASC

Lesões de tratamento preferencial (tipo C) e de tratamento de escolha (tipo D) por cirurgia aberta.

Lesões tipo C

- Estenoses múltiplas ou oclusões totalizando $15 \mathrm{~cm}$ com ou sem calcificação importante;

- Estenoses recorrentes ou oclusões que precisam tratamento após duas intervenções endovasculares.

\section{Lesões tipo D}

- Oclusões crônicas totais de artéria femoral comum ou femoral superficial (> 20 cm envolvendo a artéria poplítea);

- Oclusão total da artéria poplítea e vasos proximais da trifurcação.

TASC A

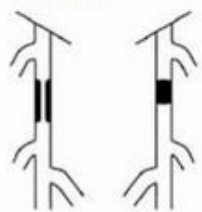

TASC B
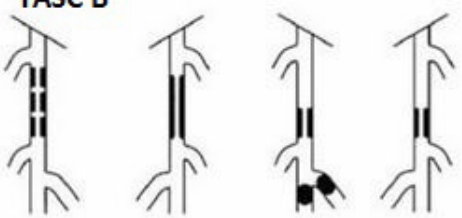

TASC C

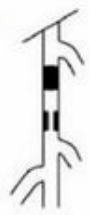

TASC D

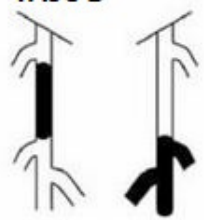

Figura 2. Classificação (esquema) das lesões infranguinais segundo o TASC II 
mento das placas ateroscleróticas na luz arterial, há tempo suficiente para que a circulação colateral possa desenvolver-se e manter o membro completamente compensado. A artéria femoral profunda é a principal via de colateralização nos membros inferiores e costuma-se dizer que é a grande responsável por manter o membro viável mesmo em situações críticas de perfusão.

Na última década a evolução sem precedentes do tratamento desses pacientes também atingiu ao território femoropoplíteo. Segundo o TASC II, a terapia endovascular é o tratamento de escolha para lesões TASC A e a cirurgia aberta é o tratamento de escolha para as lesões TASC D. Nas lesões TASC C a técnica preferida deve ser a angioplastia, e nas lesões TASC D a cirurgia (figura 2 e tabela 2). ${ }^{13}$ Ainda assim esses limites têm recebido muitas críticas desde a publicação do documento e diversos cirurgiões preconizam a técnica endovascular também para as lesões $C$ e D, sempre que possível. Enquanto não houver estudos randomizados com grandes populações, diferentes abordagens e filosofias irão coexistir. ${ }^{7}$

\section{Obstrução infrapatelar}

O tratamento da doença oclusiva infrapatelar é um dos problemas mais difíceis da cirurgia vascular. Estes pacientes possuem mais comorbidades clínicas e estão predispostos a maior risco cirúrgico e resultados ruins. $\mathrm{O}$ diabetes mellitus, que coexiste em aproximadamente $60 \%$ dos pacientes com doença infrapatelar, sobrepõe outros desafios ao tratamento cirúrgico. Além da descompensação clínica, comum pela presença de lesões complexas e infectadas e a presença de osteomielite, a calcificação excessiva dos vasos tibiais acometidos e o caráter difuso com múltiplos níveis da doença aterosclerótica reduzem as probabilidades de sucesso clínico neste território. Por fim, as opções de tratamento para esta doença são acompanhadas pela necessidade frequente de (re)intervenção.

\section{Mudança de paradigma}

Devido à complexidade clínica e cirúrgica desta doença, a indicação de intervir nesse segmento anatômico é, na imensa maioria das vezes, dedicada a prevenir a amputação do membro. Salvo raras exceções, apenas em situações de dor em repouso ou lesão trófica instalada no membro a intervenção cirúrgica está indicada. Com o avanço das técnicas endovasculares nesse território, observamos uma importante mudança de paradigma na abordagem cirúrgica desses pacientes: a filosofia de realizar o procedimento mais durável possível foi substituída por realizar intervenções menores, capazes de cicatrizar a lesão. Há pouca dúvida de que as opções endovasculares são geralmente menos duráveis do que a ponte cirúrgica aberta. Contudo, em uma coorte de pacientes idosos e de alto risco, colocou-se em xeque a durabilidade de uma intervenção versus sua invasividade e seu risco. A perviedade de longo prazo de uma reconstrução nem sempre é o fator mais relevante. O que se viu na prática é que em pacientes com úlceras isquêmicas ou gangrenas, o tratamento endovascular permite a cicatrização dessas feridas e amputações de dedos com salvamento de membro no longo prazo, mesmo se a intervenção endovascular eventualmente falhar. É bem sabido que as taxas de salvamento do membro excedem as taxas de perviedade das intervenções endovasculares. Durante a última década, testemunhamos um grande aumento no número de tratamentos endovasculares para a isquemia dos membros inferiores, e isto foi acompanhado por uma diminuição na taxa global de grande amputação.

\section{Pé diabético}

Como descrito anteriormente, os pacientes diabéticos compõem um grupo prevalente e complexo no tratamento da doença obstrutiva infrapatelar. A associação da DAOP com as lesões típicas do pé diabético impõe um desafio ainda maior ao tratamento desses pacientes. A aterosclerose é a maior causa de morte e invalidez em diabéticos, especialmente os do tipo 2. Em estudo com pacientes claudicantes, verificou-se que cerca de $43 \%$ dos indivíduos são diabéticos. A prevalência de DAOP é maior 
em pacientes diabéticos do que na população não diabética. Estima-se que 20 a $30 \%$ dos indivíduos diabéticos sejam portadores de DAOP, ainda que a prevalência real dessa associação seja difícil de ser avaliada. Esta dificuldade se deve à ausência de sintomas, mascarados pela neuropatia periférica em boa parte dos pacientes, e aos diferentes indicadores utilizados nas pesquisas epidemiológicas. ${ }^{11}$

\section{Interface na tomada de decisão}

\section{DAOP}

A colaboração entre equipe médica e time de intervenção pode ter um valioso papel em dois aspectos da rotina de tratamento de pacientes com DAOP: na avaliação dos riscos inerentes ao quadro clínico de cada paciente e suas comorbidades e na análise dos riscos e benefícios que envolvem cada opção terapêutica, inclusive seus custos e aplicabilidade para cada caso específico.

Um exemplo típico e rotineiro é a decisão de fazer ou não a estratificação do risco cardiovascular de um paciente com isquemia crítica e candidato a revascularização do membro. A realização de exames mais sofisticados encarece o preparo pré-operatório, aumenta os riscos relacionados a esses exames invasivos (a exemplo de uma coronariografia) e retarda o procedimento de revascularização do membro ampliando o risco de amputação e deterioração clínica, além de muitas vezes não estarem justificados. A mesma premissa serve para a equipe anestésica, a comunicação entre cirurgião e anestesista pode modificar a conduta anestésica ou a estratégia de intervenção e precisa ser individualizada para cada paciente.

Médicos trabalhando em conjunto e comprometidos com o resultado do tratamento são capazes de definir melhor as estratégias de abordagem para determinado paciente. Por outro lado, qualquer que seja, a intervenção representa uma agressão maior ou menor a um indivíduo já debilitado e com descompensações clínicas importantes. Muitas vezes o procedimento pode ser adiado por poucos dias para que a equipe médica possa equilibrar o paciente da melhor forma possível antes da cirurgia programada.

O Department of Health, Social Services and Public Safety do Reino Unido recomenda que todos os portadores de DAOP, como pacientes com diabetes, insuficiência renal crônica, tabagistas e idosos tenham acesso a acompanhamento dos membros inferiores por uma equipe multidisciplinar de cuidados com o pés. ${ }^{15} \mathrm{~A}$ reunião com a equipe multidisciplinar é também um valioso momento para debater e decidir entre cirurgia aberta e endovascular, embora na maioria das unidades a política de tentar primeiro a técnica endovascular esteja bem estabelecida. $^{7}$

\section{DAOP e pé diabético}

Provavelmente em nenhuma outra síndrome envolvendo a doença arterial obstrutiva periférica a adoção de um time multidisciplinar tenha tanto impacto como no caso do "pé diabético". Os mecanismos fisiopatológicos que envolvem as lesões diabéticas nos pés são multifatoriais e incluem neuropatia, infecção, isquemia e as alterações da estrutura e da biomecânica do pé. Como regra, a isquemia grave da doença arterial obstrutiva periférica requer estratégias de revascularização agressiva, mas somente essa iniciativa pode não bastar. Uma abordagem interdisciplinar agressiva deve oferecer terapia medicamentosa e cirúrgica ótimas e melhorar a evolução dessas lesões.

A última década foi prodigiosa em acumular sólidas evidências de que é possível reduzir de forma significativa as taxas de amputação de membros inferiores com a ampla adoção de duas medidas: 1) o emprego de equipes multidisciplinares que incluam médicos de diferentes especialidades, podólogos, enfermeiras, nutricionistas e assistentes sociais no acompanhamento do pé de risco e 2) educação. Nos dias de hoje é possível afirmar que até $85 \%$ das amputações poderiam ser evitadas através de um programa de cuidado ao pé diabético bem organizado, controle glicêmico adequado, educação e informação. ${ }^{16}$ 


\section{Evidências e limitações}

A criação de equipes multidisciplinares objetiva uma aferição precisa nas decisões terapêuticas. É fundamental que se realizem os protocolos de utilização de tecnologias e que se tenham, de forma clara, resultados de mortalidade e complicações da literatura para cotejamento com os dados institucionais. A utilização de banco de dados e o acompanhamento dos pacientes a longo prazo podem efetivamente ser estímulos que corroboram com a expectativa de que o tratamento por equipe é melhor. Entretanto, não há dados de literatura que embasem que as decisões tomadas por uma equipe multidisciplinar são superiores às decisões individuais. Um único estudo, não comparativo, avaliando o cenário da intervenção coronariana, mostrou que as decisões tomadas em equipe são reproduzíveis e bem fundamentadas. ${ }^{17} \mathrm{~A}$ validação científica para essa comparação se torna muito complexa, até mesmo por aspectos éticos, pois seria difícil a escolha dos participantes (equipe vs. médico isolado e paciente). Aspectos filosóficos à parte, talvez a persistência desta dúvida não seja de importância na consolidação das equipes multidisciplinares para oferecermos boas práticas clínicas aos nossos pacientes.

\section{Conclusões}

O tratamento realizado através de equipes multidisciplinares está incorporado à prática médica de maneira irreversível. A ampliação dessa modalidade, fundamentada inicialmente em pacientes com insuficiência cardíaca e doenças malignas, hoje atinge diversas indicações no campo das intervenções cirúrgicas, endovasculares e percutâneas cardiovasculares. A escolha adequada de tratamentos menos invasivos e de pacientes com maior probabilidade de bons resultados são a tônica da prática clínica. Mesmo sem a validação com base em evidências, a discussão sistemática e interdisciplinar permite de forma inequívoca a obtenção de melhores resultados, principalmente nos pacientes de alto risco. Independentemente se são candidatos ao tratamento das doenças vasculares periféricas ou cardiovasculares, o fórum de discussão na tomada de decisão está relacionado ao que se pode oferecer de melhor aos pacientes.

\section{Referências}

1. Costanzo MR, Augustine S, Bourge R, Bristow M, O'Connell JB, Driscoll D, et al. Selection and treatment of candidates for heart transplantation. A statement for health professionals from the Committee on Heart Failure and Cardiac Transplantation of the Council on Clinical Cardiology, American Heart Association. Circulation. 1995;92:3593612.

2. Serruys PW, Morice MC, Kappetein AP, Colombo A, Holmes DR, Mack MJ, et al; SYNTAX Investigators. Percutaneous coronary intervention versus coronary-artery bypass grafting for severe coronary artery disease. $\mathrm{N}$ Engl J Med. 2009;360:961-72.

3. Earnshaw JJ, Beard JD. The Vascular MDT: an essential element in the treatment of patient with vascular disease. Ann R Coll Surg Engl (Suppl). 2011;93.

4. National Institute for Heath and Clinical Excellence. Lower limb peripheral arterial disease: diagnosis and management. NICE Clinical Guideline 147. atualizada em agosto 2012, acessada em 2013 may 2. Disponível em: http://guidance.nice.org.uk/cg147.

5. Davis T. Multidisciplinary Approach to Peripheral Artery Disease. Acessadoem 2013 may 02. Disponível em: http://metrohealth.net/wpcontent/uploads/2012/01/MultidisciplinaryApproach-to-Peripheral-Vascular-Diseasedavis-back-up-Compatibility-Mode.pdf

6. The PARTNERS program: A national survey of peripheral arterial disease detection, awareness, and treatment. JAMA. 286: 2001; 1317-1324.

7. Paraskevas KI, Mikhailidis DP, Veith FJ. Comparison of te five 2011 guidelines for the treatment of carotid stenosis. J Vasc Surg. 2012;55:1504-8.

8. Department of Health and Children. Changing Cardiovascular Health. National Cardiovascular. Health Policy 2010-2019. may 2010 Published by Government Publications, Dublin. Disponível em: http://www.headway. ie/download/pdf/changing_cardiovascular health.pdf, acessado em 2013 may 02.

9. Department fo Health, Social Services and Public Safety. Service Framework for cardiovascular health and well being. Disponível em: http:// www.dhsspsni.gov.uk, acessado em 2013 may 02.

10. Selvin E, Erlinger TP. Prevalence of and risk factors for peripheral arterial disease in the 
United States: results from the National Health and nutrition Examination Survey, 999-2000. Circulation. 2004;110:738-43.

11. National Institute for Health and Care Excellence. Carotid imaging and carotid endarterectomy for people with TIA or non-disabling stroke. Disponível em: http:// pathways.nice.org.uk/pathways/stroke/ carotid-imaging-and-carotid-endarterectomyfor-people-with-tia-or-non-disabling-stroke Atualizado em 2013 jan, acessado em 2013 may 02.

12. Sumpio BE, Armstrong DG, Lavery LA, Andros G. The role of interdisciplinary team approach in the management of the diabetic foot. J Vasc Surg. 2010;51:1504-6.

13. Virgini-Magalhães CE, Bouskela E. Pé Diabético e Doença Vascular - Entre o Conhecimento Acadêmico e a Realidade Clínica. Arq Bras endocrinol Metab. 2008;52:1073-5.

14. Norgren L, Hiatt aWR, Dormandy bJA, Nehler MR, Harris KA, Fowkes FGR. Inter-Society Consensus for the Management of Peripheral Arterial Disease (TASC II). J Vasc Surg. 2007;suppl:s5A-s67A.

15. Virgini-Magalhães CE. Doença arterial obstrutiva periférica no paciente diabético: avaliação e conduta. Diretrizes 2009 Sociedade Brasileira de Diabetes, p. 33-9.

16. Murphy TP, Cutlip DE, Regensteiner JG, Mohler ER, Cohen DJ, Reynolds MR, et al. Supervised Exercise Versus Primary Stenting for Claudication Resulting From Aortoiliac Peripheral Artery Disease Six-Month Outcomes From the Claudication: Exercise Versus Endoluminal Revascularization (CLEVER) Study. Circulation. 2012;125:130.

17. Long J, Luckraz H, Thekkudan J, Maher A, Norell M. Heart team discussion in managing patients with coronary artery disease: outcome and reproducibility. Interact Cardiovasc Thorac Surg. 2012;14:594-8. 